\title{
Perfect powers in products of terms in an arithmetical progression III
}

\author{
by
}

T. N. Shorey (Bombay) and R. Tijdeman (Leiden)

1. For an integer $x>1$, we denote by $P(x)$ the greatest prime factor of $x$ and we write $\omega(x)$ for the number of distinct prime divisors of $x$. Further, we put $P(1)=1$ and $\omega(1)=0$. Let $d \geq 1, k \geq 2$ and $m \geq 0$ be integers such that $\operatorname{gcd}(m, d)=1$. Let $d_{1}, \ldots, d_{t}$ with $t \geq 2$ be distinct integers in the interval $[0, k)$. For integers $l \geq 2, y>0$ and $b>0$ with $P(b) \leq k$, we consider the equation

$$
\left(m+d_{1} d\right) \ldots\left(m+d_{t} d\right)=b y^{l} .
$$

If $P(y) \leq k$, it follows from an argument of Erdős (see [3, Lemma 2.1]) that (1) implies that

$$
t-1 \leq \frac{k \log k}{\log (m+d)}
$$

Now onward whenever we refer to equation (1), we always assume that the left-hand side of (1) has a prime factor $>k$ and thus

$$
\chi=: m+(k-1) d \geq(k+1)^{l} .
$$

Further, we write

$$
\Delta(m, k, d)=m(m+d) \ldots(m+(k-1) d) .
$$

We shall follow this notation without reference. For an account of results on equation (1), we refer to Shorey [9], Shorey and Tijdeman [12].

First, we consider equation (1) with $d=1$ which, by (2), implies that $m>k^{l}$. Erdös [2], p. 88, showed that for $\varepsilon>0$ there exists an effectively computable number $C$ depending only on $\varepsilon$ such that equation (1) with $b=1, d=1, l>2$ and $k \geq C$ implies that

$$
t \leq k-(1-\varepsilon) k \frac{\log \log k}{\log k} .
$$

Further, Erdös [1], [2], p. 88, showed that there exist effectively computable absolute constants $C_{1}$ and $C_{2}>0$ such that equation (1) with $b=1, d=1$, 
$l=2$ and $k \geq C_{1}$ implies that

$$
t \leq k-C_{2} k / \log k .
$$

See Erdős and Turk [3] for some extensions of these results. The proofs of these results are elementary. Shorey $[7,8]$ combined this elementary method of Erdös with the theory of linear forms in logarithms, irrationality measures of Baker on the approximations of certain algebraic numbers by rationals and the method of Roth-Halberstam on $\mu$-free integers to sharpen (3) considerably. It has been proved in $[7,8]$ that there exists an effectively computable absolute constant $C_{3}$ such that equation (1) with $d=1, l>2$ and $k \geq C_{3}$ implies that

$$
t \leq \nu_{l} k \quad \text { with } \quad \nu_{l}=\frac{1}{2}\left(1+\frac{4 l^{2}-8 l+7}{2(l-1)\left(2 l^{2}-5 l+4\right)}\right) .
$$

We observe that

$$
\nu_{3}=\frac{47}{56}, \quad \nu_{4}=\frac{45}{64} \quad \text { and } \quad \nu_{l} \leq \frac{299}{464}<\frac{2}{3} \quad \text { for } l \geq 5 .
$$

If $l$ is sufficiently large, estimate (5) can be sharpened. Shorey [7] applied the theory of linear forms in logarithms to prove that there exists an effectively computable absolute constant $C_{4}$ such that equation (1) with $d=1, l \geq C_{4}$ and $k \geq C_{4}$ implies that

$$
t \leq k l^{-1 / 11}+\pi(k)+2 .
$$

For $l=2$, Erdős [2], p. 88, asked whether (4) can be replaced by (3). Shorey [8] combined the method of Erdös with a theorem of Baker on the finiteness of integer solutions of the hyper-elliptic equation to show that this is, in fact, the case. More precisely, it has been shown in $[7,8]$ that given $\varepsilon>0$ there exists an effectively computable number $C_{5}$ depending only on $\varepsilon$ such that equation (1) with $d=1, l=2$ and $k \geq C_{5}$ implies (3). As a consequence of these results, we shall derive

THEOREM 1. Let $\varepsilon>0$ and $m+k-1>k^{l}$. There exists an effectively computable number $C_{6}$ depending only on $\varepsilon$ such that for $k \geq C_{6}$ we can find a prime $p$ with

$$
0<\operatorname{ord}_{p}(\Delta(m, k, 1)) \not \equiv 0(\bmod l)
$$

such that

$$
p \geq \begin{cases}(1-\varepsilon) k \log \log k & \text { if } l=2 \\ \left(1-\nu_{l}-\varepsilon\right) k \log k & \text { if } l>2 \\ (1-\varepsilon) k \log k & \text { if } l>C_{6} .\end{cases}
$$

If $m \leq k^{l}$, we observe that $\operatorname{ord}_{p}(\Delta(m, k, 1)) \not \equiv 0(\bmod l)$ for every prime $p>k$ such that $p \mid \Delta(m, k, 1)$. Therefore, it suffices to consider lower bounds 
for $P(\Delta(m, k, 1))$ to obtain an analogue of Theorem 1 in the case $m \leq k^{l}$. For this, we refer to Shorey [6] and Shorey and Tijdeman [10], Chapter 7.

Next, we consider (1) with $d>1$. There is no loss of generality in assuming that $l$ is a prime number. We put

$$
h(k)= \begin{cases}\log \log k & \text { if } l \geq 5, \\ \log \log \log k & \text { if } l=3,\end{cases}
$$

for $k>e^{e}$. We obtain the following weaker analogue of the above stated results of Shorey.

THEOREM 2. (a) Let $\varepsilon>0$. There exists an effectively computable number $C_{7}$ depending only on $\varepsilon$ and $\omega(d)$ such that equation (1) with $l=2$ and $k \geq C_{7}$ implies that

$$
t \leq k-(1-\varepsilon) k \frac{\log \log \log k}{\log k} .
$$

(b) Let $\varepsilon>0$ and $l>2$ be a prime number. There exist effectively computable numbers $C_{8}$ and $C_{9}$ depending only on $\varepsilon$ such that equation (1) with $k \geq C_{8}$ and

$$
l^{\omega(d)} \leq C_{9} k \frac{h(k)}{\log k}
$$

implies that

$$
t \leq k-(1-\varepsilon) k \frac{h(k)}{\log k} .
$$

Theorem 2 with $t=k$ is contained in Shorey and Tijdeman [12], Theorem 1. The proof of Theorem 2(a) depends on Brun's Sieve and a theorem of Evertse and Silverman [5] on the number of integer solutions of the hyperelliptic equation. The proof of Theorem 2(b) with $l \geq 5$ is elementary and we apply a theorem of Evertse [4] in the case $l=3$. We apply Theorem 2 to derive the following analogue of Theorem 1 for $\Delta(m, k, d)$.

COROLlary 1. Let $\varepsilon>0$ and $\chi>k^{l}$.

(a) There exists a prime $p$ dividing $\Delta(m, k, d)$ such that

$$
p \geq(1-\varepsilon) k \log \log \log k \quad \text { and } \operatorname{ord}_{p}(\Delta(m, k, d)) \not \equiv 0(\bmod 2)
$$

whenever $k$ exceeds an effectively computable number depending only on $\varepsilon$ and $\omega(d)$.

(b) Let $l>2$ be a prime number. There exist effectively computable numbers $C_{10}$ and $C_{11}$ depending only on $\varepsilon$ such that for $k \geq C_{10}$ and

$$
l^{\omega(d)} \leq C_{11} k \frac{h(k)}{\log k},
$$


we can find a prime $p$ dividing $\Delta(m, k, d)$ satisfying

$$
p \geq(1-\varepsilon) k h(k) \text { and } \operatorname{ord}_{p}(\Delta(m, k, d)) \not \equiv 0(\bmod l) .
$$

For an analogue of Corollary 1 in the case $\chi \leq k^{l}$, it is equivalent to considering lower bounds for $P(\Delta(m, k, d))$. We refer to Shorey and Tijdeman $[11,13]$ for these bounds. We write

$$
h^{\prime}(k)= \begin{cases}h(k) & \text { if } l \geq 3, \\ \log \log \log k & \text { if } l=2 .\end{cases}
$$

The following result is a particular case of Corollary 1 whenever $l$ and $\omega(d)$ are bounded.

Corollary 2. Let $l \geq 2$ be a prime number. Let $\varepsilon>0$ and $\chi>k^{l}$. There exists a prime $p$ dividing $\Delta(m, k, d)$ such that

$$
p \geq(1-\varepsilon) k h^{\prime}(k) \quad \text { and } \operatorname{ord}_{p}(\Delta(m, k, d)) \not \equiv 0(\bmod l)
$$

whenever $k$ exceeds an effectively computable number depending only on $\varepsilon, l$ and $\omega(d)$.

2. Proof of Theorem 2. (a) Let $c>0$ be a number depending only on $\varepsilon$ and $\omega(d)$. We shall choose $c$ later. We may assume that $k$ exceeds a sufficiently large effectively computable number depending only on $\varepsilon, c$ and $\omega(d)$. For $1 \leq i \leq t$, we see from (1) that

$$
m+d_{i} d=a_{i} x_{i}^{2}
$$

where $a_{i}$ is square-free such that $P\left(a_{i}\right) \leq k$ and $x_{i}$ is a positive integer. Let $S$ be the set of all $a_{i}$ with $1 \leq i \leq t$ such that $d_{i}>0$. We may suppose that

$$
t \geq k-(1-\varepsilon) k \frac{\log \log \log k}{\log k} .
$$

Then, we argue as in the proof of Lemma 1 of [12] to derive that

$$
\chi \geq c_{2} k^{3}(\log k)^{2} .
$$

Further, we refer to the proof of Lemma 3 of [12] to conclude from (11) and (12) that

$$
|S| \geq k-\left(1-\frac{3 \varepsilon}{4}\right) k \frac{\log \log \log k}{\log k} .
$$

Next, we follow the proof of Theorem 2 of [7] without reference. By (13), there exists a subset $S_{1}^{\prime}$ of $S$ such that $\left|S_{1}^{\prime}\right| \geq \varepsilon k / 2$ and

$$
a_{i} \leq k(\log \log k)^{1-\varepsilon / 2} \quad \text { if } a_{i} \in S_{1}^{\prime} .
$$

For $a_{i}, a_{j} \in S_{1}^{\prime}$ with $i \neq j$, the equation $x_{i}=x_{j}$ implies that

$$
\chi \leq k^{3}(\log \log k)^{1-\varepsilon / 2},
$$


by $(10),(14)$ and $\operatorname{gcd}(m, d)=1$. Consequently, we conclude from (12) and (15) that

$$
x_{i} \neq x_{j} \quad \text { if } a_{i}, a_{j} \in S_{1}^{\prime} \text { and } i \neq j .
$$

We denote by $b_{1}, \ldots, b_{s}$ all integers between $k(\log k)^{-2 c}$ and $k(\log \log k)^{1-\varepsilon / 2}$ such that every proper divisor of $b_{i}$ is less than or equal to $k(\log k)^{-2 c}$. Now, we apply Brun's Sieve to derive that

$$
s \leq k(\log \log k)^{-\varepsilon / 2} .
$$

We write $S_{2}^{\prime}$ for the set of all $a_{i} \in S_{1}^{\prime}$ with $a_{i} \geq k(\log k)^{-2 c}$. Then $\left|S_{2}^{\prime}\right| \geq$ $\varepsilon k / 4$. Further, we observe that every element of $S_{2}^{\prime}$ is divisible by at least one $b_{i}$. Let $S_{3}^{\prime}$ be the maximal subset of $S_{2}^{\prime}$ such that every $b_{i}$ appears in the factorisation of at most two elements of $S_{3}^{\prime}$. Then

$$
\left|S_{3}^{\prime}\right| \leq 2 s \leq 2 k(\log \log k)^{-\varepsilon / 2} .
$$

We write $S_{4}^{\prime}$ for the complement of $S_{3}^{\prime}$ in $S_{2}^{\prime}$. If $a_{i}=a_{i_{1}} \in S_{4}^{\prime}$, there exist $a_{i_{2}}, a_{i_{3}} \in S_{3}^{\prime}$ and $b_{\nu}$ such that $a_{i_{1}}, a_{i_{2}}$ and $a_{i_{3}}$ are divisible by $b_{\nu}$. Also, we observe that $\left|S_{4}^{\prime}\right| \geq \varepsilon k / 8$. We put

and

$$
B_{1}=b_{\nu}^{-1} a_{i_{1}}, \quad B_{2}=b_{\nu}^{-1} a_{i_{2}}, \quad B_{3}=b_{\nu}^{-1} a_{i_{3}}
$$

$$
R=b_{\nu}^{-1}\left(d_{i_{2}}-d_{i_{1}}\right), \quad R^{-1}=b_{\nu}^{-1}\left(d_{i_{3}}-d_{i_{1}}\right) .
$$

Since $\operatorname{gcd}(m, d)=1$, we observe that $B_{1}, B_{2}, B_{3}, R$ and $R^{\prime}$ are integers of absolute values not exceeding $(\log k)^{3 c}$. By $(10)$, we have

$$
B_{2} B_{3}\left(d^{-1} x_{i_{2}} x_{i_{3}}\right)^{2}=\left(B_{1}\left(\frac{x_{i}}{\sqrt{d}}\right)^{2}+R\right)\left(B_{2}\left(\frac{x_{i}}{\sqrt{d}}\right)^{2}+R^{\prime}\right) .
$$

There are at most $32(\log k)^{15 c}$ of these equations. Now, we apply Theorem 1 of Evertse and Silverman [5], $\left|S_{4}^{\prime}\right| \geq \varepsilon k / 8$ and (16) to conclude that

$$
\log k \leq(\log k)^{c c^{\prime}}
$$

where $c^{\prime}$ is an effectively computable number depending only on $\varepsilon$ and $\omega(d)$. Let $c=\left(2 c^{\prime}\right)^{-1}$ to notice that (17) is not possible.

(b) We do not give the details, since they are similar to the proof of Corollary 1 of [12]. See Remark (ii) of [12], p. 343.

3. Let $\varepsilon>0$. We suppose that $k$ exceeds a sufficiently large effectively computable number depending only on $\varepsilon$. Let $F(k)$ be a non-decreasing function of $k$ such that $F(k)>k$. We write

$$
T(d)=\{m, m+d, \ldots, m+(k-1) d\} .
$$

Let $T^{\prime}(d)$ be the subset of $T(d)$ of all elements $m+i d$ satisfying $P(m+i d) \leq$ $k$. For every prime $q$ with $k<q<F(k)$, we let $i_{q} \in[0, q)$ be such that 
$m+i_{q} d \equiv 0(\bmod q)$ (if such an $i_{q}$ exists). We denote by $T(d, F(k))$ the subset of $T(d)$ obtained by deleting $m+i_{q} d$ for all primes $q$ satisfying $k<$ $q<F(k)$. We put

$$
|T(d, F(k))|=t(d, k)=t
$$

and we write

$$
T(d, F(k))=\left\{m+d_{1} d, \ldots, m+d_{t} d\right\}
$$

where $d_{1}, \ldots, d_{t}$ are distinct integers in the interval $[0, k)$. We observe that

$$
t \geq k-\pi(F(k)) \text {. }
$$

The following lemma is useful for the proof of Theorem 1 and Corollary 1.

LEMMA 1. Let $\varepsilon>0$ and $\chi>k^{l}$. There exists an effectively computable number $C_{12}$ depending only on $\varepsilon$ such that for $k \geq C_{12}$, we have

$$
\left|T^{\prime}(d)\right| \leq\left(l^{-1}+\varepsilon\right) k .
$$

Proof. Let $\varepsilon_{1}=\varepsilon / 2$. For $\varepsilon_{1} k \leq i<k$, we observe that

$$
m+i d \geq i \chi /(k-1) \geq \varepsilon_{1} \chi \geq \varepsilon_{1} k^{l} .
$$

Now, we apply Lemma 2 of [11] with $T$ the set of all $m+i d$ such that $i \geq \varepsilon_{1} k$ and $P(m+i d) \leq k$. Then

$$
\left|T^{\prime}(d)\right| \leq \frac{k \log k}{\log \left(\varepsilon_{1} k^{l}\right)}+\varepsilon_{1} k+\pi(k) \leq\left(l^{-1}+\varepsilon\right) k .
$$

Proof of Theorem 1 . Put $d=1$. We may assume that $k \geq c_{1}$ where $c_{1}$ is a sufficiently large effectively computable number depending only on $\varepsilon$. We shall apply the results of Shorey on equation (1) with $d=1$ stated in the beginning of Section 1 without reference in the proof of Theorem 1.

Suppose $l=2$. Put $F(k)=(1-\varepsilon) k \log \log k$. By (19) and Prime Number Theorem, we observe that

$$
t>k-\left(1-\frac{\varepsilon}{2}\right) k \frac{\log \log k}{\log k} \geq 2 .
$$

By (21),(20) and (18), we derive that there exists an $i$ with $1 \leq i \leq t$ such that $m+d_{i}$ is divisible by a prime $>k$. Furthermore, we may suppose that equation (1) with $d=1, l=2$ and $k \geq c_{1}$ is satisfied. Therefore, inequality (3) with $\varepsilon$ replaced by $\varepsilon / 2$ is valid. This contradicts (21).

Next suppose $l>2$. Put $F(k)=\left(1-\nu_{l}-\varepsilon\right) k \log k$. Then, we derive from (19), (5) and (20) that

$$
t>\left(\nu_{l}+\frac{\varepsilon}{2}\right) k>\left(l^{-1}+\varepsilon\right) k \geq\left|T^{\prime}(1)\right| .
$$

Consequently, we may suppose that (1) with $d=1, l>2$ and $k \geq c_{1}$ is satisfied. Hence, we conclude (5), which contradicts (22). 
Now suppose that $l \geq c_{1}$. Put $F(k)=(1-\varepsilon) k \log k$. Then

$$
\left|T^{\prime}(1)\right| \leq\left(l^{-1}+\frac{\varepsilon}{4}\right) k<\frac{\varepsilon k}{2} \leq t
$$

whenever $c_{1}$ is sufficiently large. Therefore, we may assume that (1) with $d=1, k \geq c_{1}$ and $l \geq c_{1}$ is satisfied. Hence, we conclude (6), which contradicts (23) if $c_{1}$ is sufficiently large.

Proof of Corollary 1. (a) We may assume that $k$ exceeds a sufficiently large effectively computable number $c_{2}$ depending only on $\varepsilon$ and $\omega(d)$. Put $F(k)=(1-\varepsilon) k \log \log \log k$. Then

$$
t>k-\left(1-\frac{\varepsilon}{2}\right) k \frac{\log \log \log k}{\log k}
$$

and we may suppose that equation (1) with $l=2, k \geq c_{2}$ is satisfied. On the other hand, we apply Theorem $2($ a) to conclude (7) with $\varepsilon$ replaced by $\varepsilon / 2$. This is a contradiction.

(b) We may assume that $C_{11} \leq C_{9}$ so that (9) implies (8). Now, we take $F(k)=(1-\varepsilon) k h(k)$ to argue as above for deriving Corollary 1 (b) from Theorem 2(b).

\section{References}

[1] P. Erdős, Note on the product of consecutive integers (I), J. London Math. Soc. 14 (1939), 194-198.

[2] - On the product of consecutive integers III, Indag. Math. 17 (1955), 85-90.

[3] P. Erdös and J. Turk, Products of integers in short intervals, Acta Arith. 44 (1984), 147-174.

[4] J.-H. Evertse, On the equation $a x^{n}-b y^{n}=c$, Compositio Math. 47 (1982), 289315.

[5] J.-H. Evertse and J. H. Silverman, Uniform bounds for the number of solutions to $Y^{n}=f(X)$, Math. Proc. Cambridge Philos. Soc. 100 (1986), 237-248.

[6] T. N. Shorey, On gaps between numbers with a large prime factor II, Acta Arith. 25 (1974), 365-373.

[7] —, Perfect powers in values of certain polynomials at integer points, Math. Proc. Cambridge Philos. Soc. 99 (1986), 195-207.

[8] - , Perfect powers in products of integers from a block of consecutive integers, Acta Arith. 49 (1987), 71-79.

[9] - Some exponential diophantine equations, in: New Advances in Transcendence Theory, A. Baker (ed.), Cambridge University Press, 1988, 217-229.

[10] T. N. Shorey and R. Tijdeman, Exponential Diophantine Equations, Cambridge Tracts in Math. 87, Cambridge University Press, 1986.

[11] - - - On the greatest prime factor of an arithmetical progression II, Acta Arith. 53 (1990), 499-504.

[12] - , - Perfect powers in products of terms in an arithmetical progression, Compositio Math. 75 (1990), 307-344. 
[13] T. N. Shorey and R. Tijdeman, On the greatest prime factor of an arithmetical progression III, in: Diophantine Approximation and Transcendental Numbers, Luminy 1990, Ph. Philippon (ed.), to appear.

SCHOOL OF MATHEMATICS

TATA INSTITUTE OF FUNDAMENTAL RESEARCH

HOMI BHABHA ROAD

BOMBAY 400005, INDIA
MATHEMATICAL INSTITUTE

R. U. LEIDEN

P.O. BOX 9512

2300 RA LEIDEN, THE NETHERLANDS 